\title{
The direct binding of collagen XVII and collagen IV is disrupted by pemphigoid autoantibodies
}

\author{
Mayumi Kamaguchi ${ }^{1,2} \cdot$ Hiroaki Iwata $^{1} \cdot$ Wataru Nishie $^{1} \cdot$ Ellen Toyonaga $^{1} \cdot$ Hideyuki Ujiie $^{1} \cdot K^{2}$ Natsuga $\mathbb{D}^{1} \cdot$ \\ Yoshimasa Kitagawa ${ }^{2} \cdot$ Hiroshi Shimizu ${ }^{1}$
}

Received: 15 May 2018 / Revised: 11 June 2018 / Accepted: 9 July 2018 / Published online: 8 August 2018

(c) United States \& Canadian Academy of Pathology 2018

\begin{abstract}
The basement membrane zone (BMZ) is framed by hemidesmosomes and extracellular matrix (ECM) including collagen IV (COL4). Hemidesmosomes are multiprotein complexes that include collagen XVII (COL17). BMZ proteins can be targeted in autoimmune subepidermal blistering diseases, e.g., pemphigoid targeting COL17. The blistering mechanisms in pemphigoid have not been fully elucidated, especially in mucous membrane pemphigoid (MMP), which mainly affects the mucosa. In this study, we showed that oral lesions in pemphigoid may be attributed to the inhibition of protein-protein interactions by autoantibodies. Using immunoprecipitation, we revealed that COL17 directly binds to COL4 in normal human keratinocytes and normal human oral keratinocytes. In particular, the C-terminus of COL17 is binding site to COL4 in oral keratinocytes. The precise COL4-binding region on COL17 was determined by protein-protein binding assay to be from amino acid $\mathrm{Gly}^{1175}$ to $\mathrm{Asp}^{1340}$ on the C-terminus. MMP-IgG or mAb recognizing the C-terminus hindered the interaction of COL17 with COL4 in oral keratinocytes. Furthermore, keratinocyte adhesion strength to COL4-coated plates was significantly reduced by the treatment of $\mathrm{mAb}$ against the C-terminus. In addition, the inflammatory infiltrates around perilesions were significantly less in MMP compared to BP. These results indicate that pemphigoid IgG targeting the C-terminus plays a pathogenic role in blister formation in the oral mucosa to inhibit protein interactions with less inflammation.
\end{abstract}

\section{Introduction}

The basement membrane zone (BMZ) is located beneath epithelial structures and framed by hemidesmosomes and extracellular matrix (ECM). Hemidesmosomes are multiprotein complexes that include BP230, CD151, plectin, integrin $\alpha 6 \beta 4$, and collagen XVII (COL17, also called BP180) [1, 2]. Transmembrane COL17 interacts

Electronic supplementary material The online version of this article (https://doi.org/10.1038/s41374-018-0113-9) contains supplementary material, which is available to authorized users.

$\triangle$ Hiroaki Iwata

hiroaki.iwata@med.hokudai.ac.jp

1 Department of Dermatology, Hokkaido University Graduate School of Medicine, Kita-ku, Sapporo 060-8638, Japan

2 Department of Oral Diagnosis and Medicine, Hokkaido University Graduate School of Dental Medicine, Kita-ku, Sapporo 060-8638, Japan extracellularly with integrin $\alpha 6$ and laminin 332 and intracellularly with BP230, plectin, and integrin $\beta 4$ [3-10]. Together with hemidesmosomes, ECM, which is mainly composed of collagen IV (COL4), is critical for the maintenance of tissue architecture and functions.

The defects in BMZ proteins induce epidermolysis bullosa, which is a group of congenital skin-blistering disorders [11, 12]. In addition, BMZ proteins can be targeted in several autoimmune blistering diseases. Bullous pemphigoid (BP), which mainly presents as skin blisters or erosions on the whole body, targets COL17 and/or BP230 [13, 14]. Mucous membrane pemphigoid (MMP), which predominantly presents as mucosal lesions, including on the oral mucosa, mainly targets COL17, laminin 332, and integrin $\alpha 6 \beta 4$ [15].

The precise blistering mechanisms induced by autoantibodies have been well studied and include the direct inhibition of protein-protein binding by autoantibodies in pemphigus (steric hindrance) [16, 17] or Fc-mediated complement and inflammatory cell activation in pemphigoid $[18,19]$. Steric hindrance induces the detachment of 
the protein-protein interactions by autoantibodies without inflammation. Therefore, the blistering mechanisms in pemphigus and pemphigoid are thought to be distinct, especially regarding inflammation.

Differences in the major targeted epitopes on COL17 are reported in $\mathrm{BP}$ and MMP: the non-collagenous (NC) 16A domain in BP [20] and the C-terminus in MMP [21]. However, it remains unclear how autoantibodies targeting different epitopes induce diverse clinical manifestations. Recently, we reported the different expression levels of COL17 in the skin and oral mucosa [22]. The high amount of COL17 in the oral mucosa compensates for its depletion induced by BP-IgG. Furthermore, immunoglobulin G (IgG) against the C-terminus of COL17, which is thought to be non-pathogenic, may have pathogenicity in cooperation with $\operatorname{IgG}$ against NC16A, which is pathogenic. This evidence explains the predominant skin lesions in BP. In addition, it has been reported that BP patients showing non-inflammatory phenotype with few erythematous skin lesions frequently induced by dipeptidyl peptidase-4 inhibitors (DDP-4i) [23] are associated with HLA-DQB1*03:01 [24], which has also been identified in MMP [25]. Interestingly, autoantibodies from these noninflammatory BP patients or COL17-type MMP patients mainly target the middle portion or C-terminus of COL17 $[21,23]$. However, the precise pathogenicity of autoantibodies toward regions outside of NC16A (non$\mathrm{NC} 16 \mathrm{~A})$ is unclear.

In this study, we found that MMP patients tended to have reduced inflammatory findings in histological analysis. We hypothesized that one of the pathogenic mechanisms for oral lesions in pemphigoid might be related to the inhibition of the protein-protein interactions by autoantibodies. We focused on the interaction of COL17 with ECM proteins, especially with COL4. We found direct binding between COL4 and COL17 in normal epidermal and oral keratinocytes. In particular, this binding is disrupted by IgG against the C-terminus in oral keratinocytes.

\section{Materials and methods}

\section{Total IgG purification from BP or MMP patients}

MMP patients fulfilled the following inclusion criteria; (i) clinical blistering or erosion on the oral mucosa, and (ii) linear deposits of $\mathrm{IgG}$ and/or C3 at the BMZ by direct immunofluorescence (DIF). BP patients fulfilled the following inclusion criteria: (i) clinical blistering or erosion on the skin, (ii) linear deposits of IgG and/or C3 at the BMZ as determined by DIF, and (iii) circulating autoantibodies against COL17 as detected by COL17-NC16A enzyme- linked immunosorbent assay/chemiluminescence enzyme immunoassay (ELISA/CLEIA) (MBL, Nagoya, Japan). Non-NC16A BP patients fulfilled the following inclusion criteria (i) clinical blistering or erosion on the skin, (ii) linear deposits of $\operatorname{IgG}$ and/or C3 at the BMZ by DIF, and (iii) circulating autoantibodies detected by full-length COL17 ELISA [23] but not by COL17-NC16A ELISA/ CLEIA (MBL, Nagoya, Japan). DIF, indirect immunofluorescence (IIF), $1 \mathrm{M} \mathrm{NaCl}$ split skin IIF, and COL17 ELISA were performed as previously described [23, 26, 27]. Total IgG was purified using a protein $G$ affinity column according to the manufacturer's instructions (GE Healthcare, Amersham, UK) (final concentrations: $20 \mathrm{mg}$ / ml). In accordance with the Hokkaido University Hospital bylaws and standard operating procedures approved by the Hokkaido University Hospital Review Board, we obtained written consent for experimental procedures to be performed. The studies were conducted in accordance with the Helsinki guidelines.

\section{Cell culture}

Primary normal human oral mucosal keratinocytes (NHOMKs) and normal human epidermal keratinocyte (NHEKs) were generated from normal healthy volunteers as previously described [22].

\section{Production of recombinant COL17}

Full-length human COL17 (NM-000292, Met $^{1}$ to Pro ${ }^{1497}$ ) and two different C-terminal deleted forms, COL $17 \Delta 1$ $\left(\mathrm{Met}^{1}\right.$ to $\mathrm{Asp}^{1340}$ ) and $\mathrm{COL} 17 \Delta 2\left(\mathrm{Met}^{1}\right.$ to $\left.\mathrm{Arg}^{1174}\right)$, were produced as previously reported [5, 28] (Fig. 2a).

\section{Hematoxylin and eosin (H\&E) staining and inflammatory cell counts}

The perilesional skin or oral mucosa was obtained from BP or MMP patients, respectively. H\&E staining was performed as previously described [22]. To measure total infiltrating cells or eosinophils, the number of cells was counted in perilesions. A $220 \times 180 \mu \mathrm{m}^{2}$ under $\times 400$ magnification with a high-power field was studied to count the average number of infiltrating cells in BP and MMP.

\section{Immunoprecipitation}

NHEKs and NHOMKs were lysed with $1 \%$ octylphenoxypolyetheanol (Nonidet P-40, Caledon Laboratories Ltd, Georgetown, Canada) containing protease inhibitor cocktails (Sigma Aldrich, St. Louis, MO). Anti-human COL17 monoclonal antibody (mAb) targeting COL17-NC16A 
(TS39-3) [29], anti-human COL17 mAb targeting the Cterminus of COL17 (C17-C1), anti-DDDDK mAb (M2, Sigma Aldrich, St Louis, MO), anti-human COL $4 \alpha 1 \mathrm{Ab}$ (PHM-12 + CIV22), or mIgG1 (Sigma Aldrich, St. Louis, MO) $(10 \mu \mathrm{g})$ was diluted in $200 \mu \mathrm{l}$ of phosphate-buffered saline (PBS) and then incubated with $25 \mu$ protein $G$ coupled Dynabeads (Thermo Fisher Scientific, Rockford, IL) for $1 \mathrm{~h}$ at room temperature. Then $100 \mu$ cell lysates were diluted in $400 \mu \mathrm{l}$ of PBS and incubated with Dynabeads-Ab complex overnight at $4{ }^{\circ} \mathrm{C}$. After washing with PBS, sample were boiled for $5 \mathrm{~min}$ at $95^{\circ} \mathrm{C}$.

\section{Immunoblotting}

Preparation of cell lysates, sodium dodecyl sulfatepolyacrylamide gel electrophoresis, and transfer to nitrocellulose membrane were performed as previously described [22].

For blotting, the membranes were incubated with antihuman COL17 mAb targeting COL17-NC16A (TS39-3, final concentration $2 \mu \mathrm{g} / \mathrm{ml}$ ), anti-human COL17 mAb targeting the C-terminus of COL17 (C17-C1, final concentration $2 \mu \mathrm{g} / \mathrm{ml}$ ) [30], anti-human COL17 Ab (09040, final concentration $2 \mu \mathrm{g} / \mathrm{ml}$ ) [29], anti-human COL4 $\alpha 1 \mathrm{Ab}$ (Collagen IV alpha 1, 1:500, Novos Biologicals, Littleton, CO.), anti-DDDDK-tag mAb (M2, 1:1000, Sigma Aldrich, St Louis, MO), anti-human laminin $332 \mathrm{Ab}$ (laminin5, 1:500, Abcam, Cambridge, UK), anti-human integrin $\alpha 6 \mathrm{Ab}$ (ITGA6, 1:500, Sigma-Aldrich, St. Louis, MO), anti-human integrin $\beta 4 \mathrm{Ab}(\mathrm{H}-101,1: 500$, Santa Cruz Biotechnology, Dallas, TX), and anti-human $\beta$-tubulin Ab (H-235, 1:1000, Santa Cruz Biotechnology, Dallas, TX) with $2 \%$ skimmed milk in TBS/T overnight at $4{ }^{\circ} \mathrm{C}$. Horseradish peroxidase (HRP)-conjugated anti-mouse or anti-rabbit IgG (1:5000, Life Technologies, Carlsbad, CA) in TBS/T was reacted for $1 \mathrm{~h}$ at room temperature. Signals were visualized with Clarity Western ECL Substrate (Bio-Rad Laboratories, Hercules, CA).

\section{In vitro binding test between COL17 and COL4}

The 96-well plates (Thermo Fisher Scientific, Rockford, IL) were coated with $500 \mathrm{ng} /$ well of human COL4 protein (Sigma Aldrich, St. Louis, MO) in $50 \mathrm{mM}$ carbonate buffer pH 9.5 overnight at $4{ }^{\circ} \mathrm{C}$. Non-specific binding was reduced by blocking plates with $2 \%$ bovine serum albumin (BSA) in PBS for $1 \mathrm{~h}$ at room temperature. Human COL17 recombinant proteins were added at $500 \mathrm{ng} /$ well and incubated for $2 \mathrm{~h}$. To quantify COL 17 binding to COL4, full-length human COL17 recombinant protein was used with serial dilutions, and the standard curve was generated. After extensive washing, the plates were incubated with HRP-conjugated anti-DDDDK mAb (M2, 1:20,000, Sigma Aldrich, St. Louis, MO). After another washing, the enzyme substrate solution containing 3, 3', 5, 5'-tetramethylbenzidine was added to each well. The enzyme reaction was allowed to proceed for $20 \mathrm{~min}$ in the dark, at which point the color reaction was stopped by adding $0.12 \mathrm{~N}$ hydrochloric acid $(\mathrm{HCl})$. The absorbance was measured at $450 \mathrm{~nm}$ with the correlation wavelength set at $620 \mathrm{~nm}$ by a microplate reader (TECAN Austria $\mathrm{GmbH})$. To evaluate the binding disturbance between COL17 and COL4, COL17 recombinant proteins (250 ng/ well) were preincubated with mAbs TS39-3 or C17-C1 (250 ng/well), MMP-IgG, or BP-IgG (20 $\mu \mathrm{g} /$ well) for $1 \mathrm{~h}$ at room temperature and then added to the plates. To quantify COL17 binding to COL4, full-length human COL17 recombinant protein was used with serial dilutions, and the standard curve was generated. After blocking with $2 \%$ BSA in PBS for $1 \mathrm{~h}$ at room temperature, the plates were incubated with HRP-conjugated antiDDDDK mAb (M2, 1:20,000, Sigma Aldrich, St. Louis, MO). The enzyme reaction was allowed to proceed for $20 \mathrm{~min}$. The absorbance was measured at $450 \mathrm{~nm}$ with the correlation wavelength set at $620 \mathrm{~nm}$ by a microplate reader (TECAN Austria $\mathrm{GmbH}$ ).

\section{Competitive COL17-ELISA}

To investigate whether MMP-IgG had an identical epitope with mAb C17-C1, we performed competitive COL17ELISA. The 96-well plates (Thermo Fisher Scientific, Rockford, IL) were coated with $250 \mathrm{ng} /$ well of human full-length COL17 in $50 \mathrm{mM}$ carbonate buffer $\mathrm{pH} 9.5$ overnight at $4{ }^{\circ} \mathrm{C}$. After blocking with $2 \%$ BSA in PBS, the plates were incubated with MMP-IgG, BP-IgG, or normal human IgG $(20 \mu \mathrm{g} /$ well $)$ for $1 \mathrm{~h}$. After extensive washing, mAbs TS39-3 or C17-C1 (40 ng/well) was incubated, followed by HRP-conjugated anti-mouse IgG (1:20,000, Sigma Aldrich, St. Louis, MO). The enzyme reaction was allowed to proceed for $20 \mathrm{~min}$. The absorbance was measured at $450 \mathrm{~nm}$ with the correlation wavelength set at $620 \mathrm{~nm}$ by a microplate reader (TECAN Austria $\mathrm{GmbH}$ ).

\section{Cell adhesion test to COL4 coated plate}

Cell adhesion test were performed as described previously with minor modifications [31]. Briefly, 12-well plates were coated with human COL4 (Cell matrix type IV collagen, NITTA, Osaka, Japan) with $1 \mathrm{mM}$ of $\mathrm{HCl}$. NHOMKs were seeded in plates and cultured in CnT-Prime (CELLnTEC, Bern Switzerland) for $24 \mathrm{~h}$. The medium was changed to Dulbecco's modified Eagle's medium (DMEM) (Life 
a)

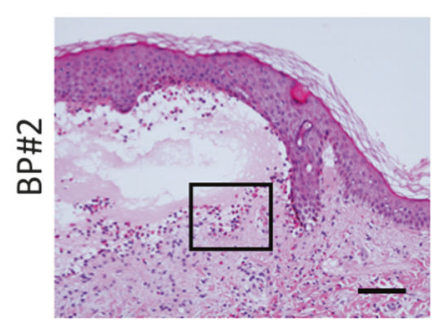

BP

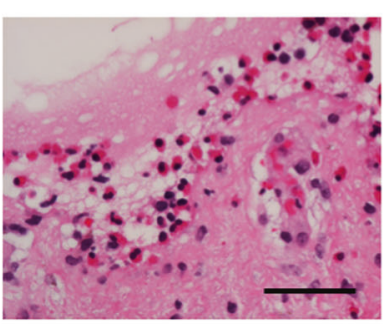

MMP
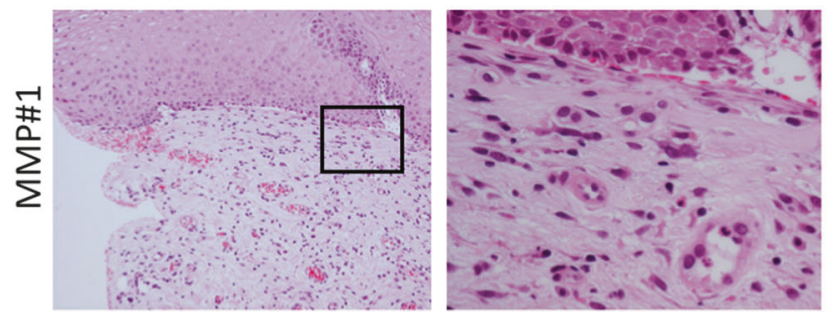

b)
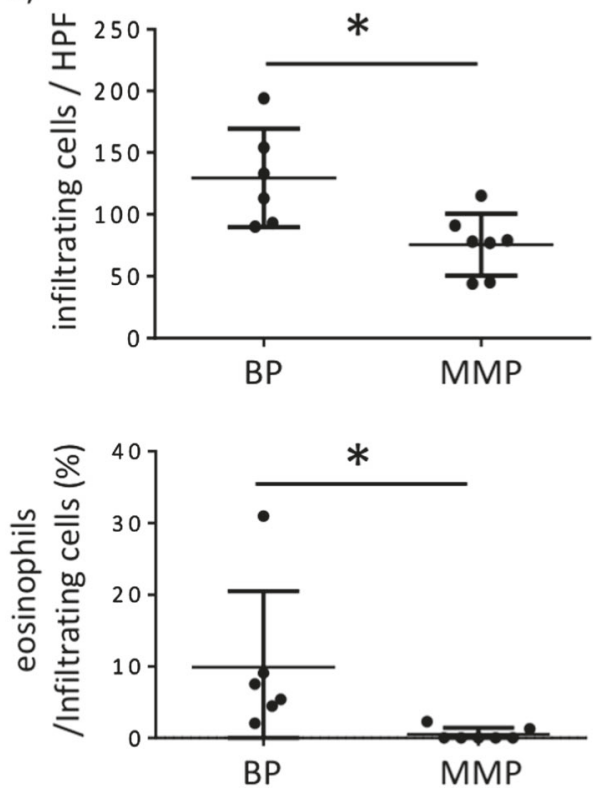

Fig. 1 Oral lesions in MMP are less inflammatory than skin lesion in BP. a Representative histopathological findings of typical BP patients with autoantibodies against NC16A and MMP are shown. H\&E staining, scale bar $=100 \mu \mathrm{m}$ (left). The black square indicates the area of $220 \times 180 \mu \mathrm{m}^{2}$ under $\times 400$ magnification high-power field (HPF),

Technologies, Tokyo, Japan) containing $1.8 \mathrm{mM}$ of calcium simultaneously with mAb C17-C1 or mIgG1 $(2.5 \mu \mathrm{g} / \mathrm{ml})$ and cultured for $12 \mathrm{~h}$. After vibration stress, the remaining cells on the plates were counted.

\section{Treatment of cultured keratinocytes with mAbs against COL17}

To investigate COL17 depletion, NHOMKs were treated with $\mathrm{mAbs}$ as previously reported, with some modifications [31]. Briefly, NHOMKs were cultured to approximately 60-70\% confluence for $24 \mathrm{~h}$ in CnT-Prime (CELLnTEC, Bern Switzerland). Medium was changed to DMEM (Life Technologies, Tokyo, Japan), containing $1.8 \mathrm{mM}$ calcium simultaneously with mAb C17-C1 or mIgG1 $(2.5 \mu \mathrm{g} / \mathrm{ml})$, and cultured for $12 \mathrm{~h}$. The cells were lysed with RIPA buffer (Thermo Fisher Scientific, Rockford, IL) containing protease inhibitor cocktails (Sigma Aldrich, St. Louis, MO) and then performed immunoblotting.

\section{Statistical analysis}

Graph Pad PRISM software Version 7.0 was used to analyze the quantitative data. $p$ Values were determined using Student's $t$ test or one-way analysis of variance followed by Tukey's test. $p<0.05$ was considered statistically significant. scale bare $=50 \mu \mathrm{m}$ (right). b Comparison of the total number of infiltrating cells (upper) and percentage of eosinophils (lower) between $\mathrm{BP}(n=6)$ and MMP $(n=7)$. The data are expressed as the mean $\pm \mathrm{S}$. D. Student's $t$ test. $* 0.01<p<0.05$

\section{Results}

\section{Oral lesions in MMP are less inflammatory than skin lesion in BP}

First, we evaluated the histopathological findings at lesional areas in BP and MMP. Patient information is summarized in Supplementary Table 1. MMP showed significantly fewer inflammatory cell infiltrations, including eosinophils, compared to BP (Fig. 1a, b). These findings indicated that the blisters occur with less inflammation in MMP.

\section{mAb targeting C-terminus (C17-C1) did not co- precipitate COL4}

Next, we investigated the interaction of COL17 with COL4. We precipitated COL17 and COL4 in cell lysates of NHEKs and NHOMKs by two mAbs targeting human $\mathrm{COL} 17, \mathrm{mAb} \mathrm{C} 17-\mathrm{C} 1$ targeting the C-terminus and $\mathrm{mAb}$ TS39-3 targeting NC16A (Fig. 2a). mAbs TS39-3 and C17-C1 co-precipitated COL4 with COL17 in NHEK lysates (Fig. 2b). In NHOMK lysates, COL17 and COL4 were clearly detected with precipitation using mAb TS393 (Fig. 2c). However, when NHOMK cell lysate was incubated with $\mathrm{mAb} \mathrm{C} 17-\mathrm{C} 1, \mathrm{COL} 4$ was not detected (Fig. 2c). The anti-COL4 antibody co-precipitated COL17 
Fig. $2 \mathrm{mAb}$ targeting $\mathrm{C}$ -

terminus (C17-C1) did not coprecipitate COL4. a Schema of full-length human COL17 and recombinant $\mathrm{C}$-terminal-deleted COL17 (COL17 $\Delta 1, \operatorname{COL} 17 \Delta 2)$ expressed by HEK 293 cells. The epitopes of mAb TS39-3

$\left(\mathrm{Asp}^{552}-\mathrm{Gln}^{545}\right)$ and mAb C17C1 $\left(\right.$ Gly $^{1316}$-Asp ${ }^{1340}$ )

are indicated.

b Immunoprecipitation with mAbs TS39-3, C17-C1, antihuman $\mathrm{COL} 4 \mathrm{~A} 1 \mathrm{Ab}$, or $\mathrm{mIgG} 1$ using cell lysates of NHOMK. Blotting was performed using anti-human COL17 Ab or antihuman COL4A1 Ab. The protein amounts in the cell lysates were analyzed by immunoblotting as input (10\% amount of IP). c

Immunoprecipitation with $\mathrm{mAbs}$ TS39-3, C17-C1, or mIgG1 using cell lysates of NHOMK. d Immunoblotting was performed using anti-human laminin 332, anti-human integrin $\alpha 6$, or anti-human integrin $\beta 4$. IP $(\mathrm{Ab})$

immunoprecipitation antibody, IB (Ab) immunoblotting antibody a)

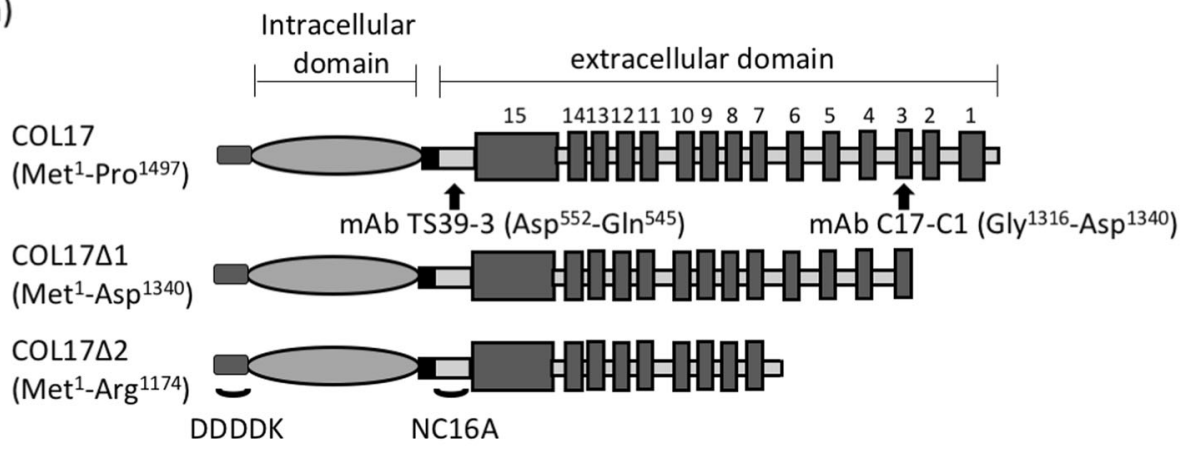

b)

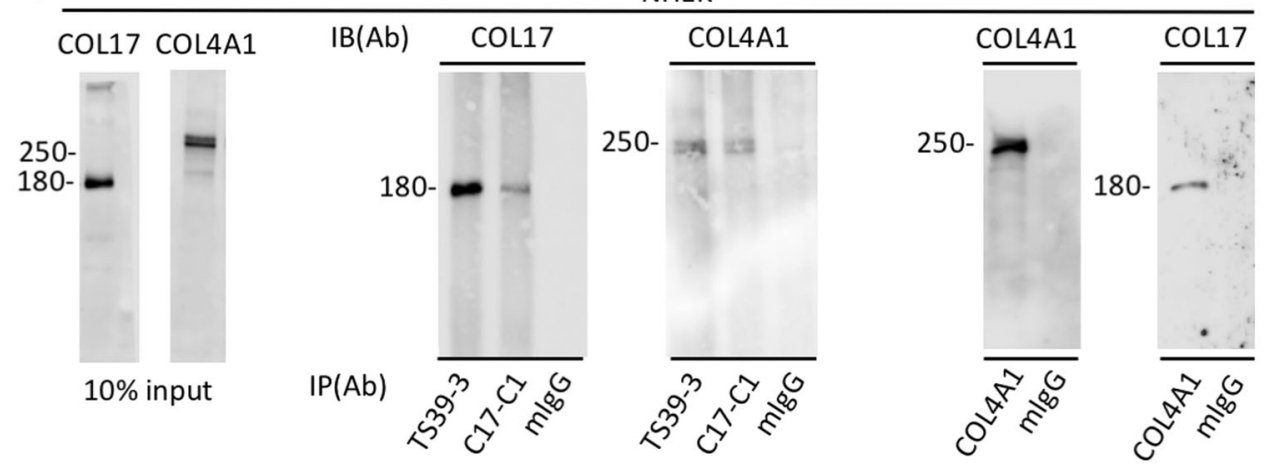

c)

NHOMK
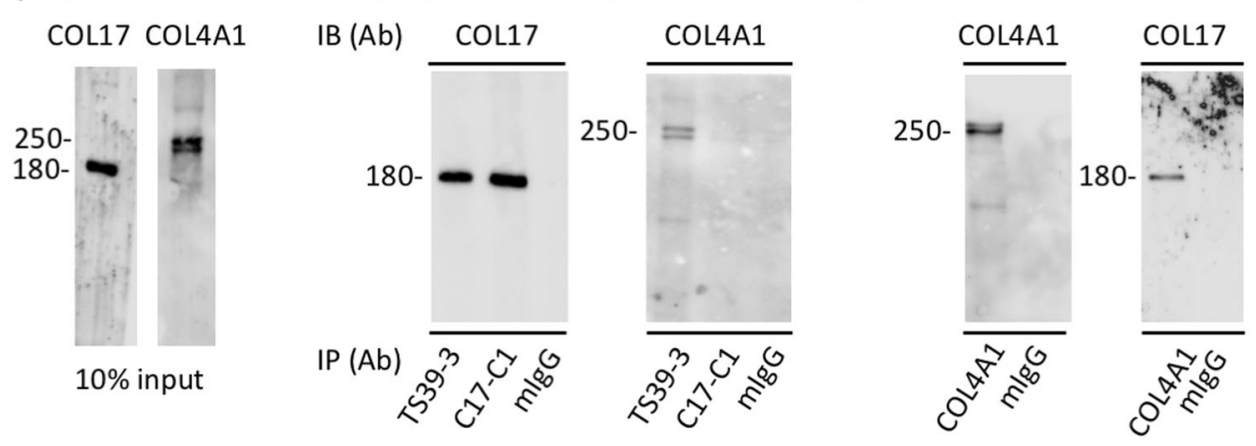

d)
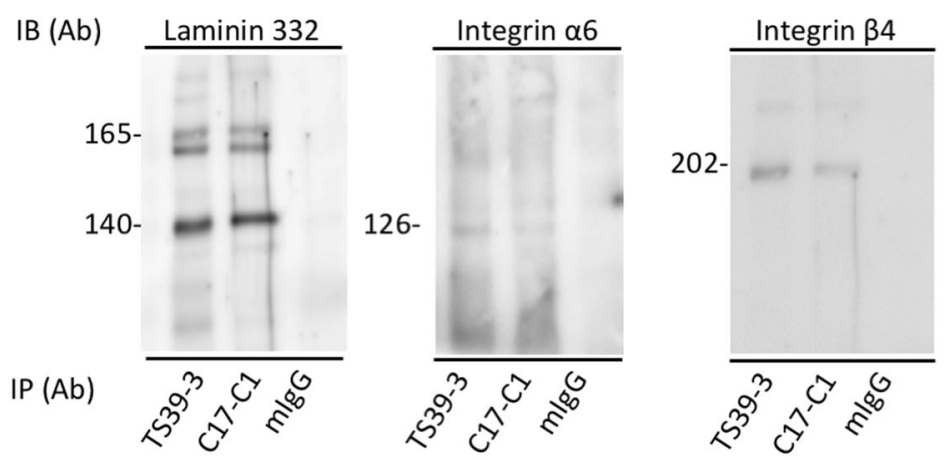

in both NHEK and NHOMK lysates (Fig. 2b, c). Both laminin 332 and integrin $\alpha 6 / \beta 4$ were detected when mAbs TS39-3 or C17-C1 were used for the precipitation (Fig. 2d). These results indicated that COL17 directly interacts with COL4. However, the binding region of COL17 and COL4 may differ between NHEKs and NHOMKs and be hindered by mAb $\mathrm{C} 17-\mathrm{C} 1$ in NHOMKs. 

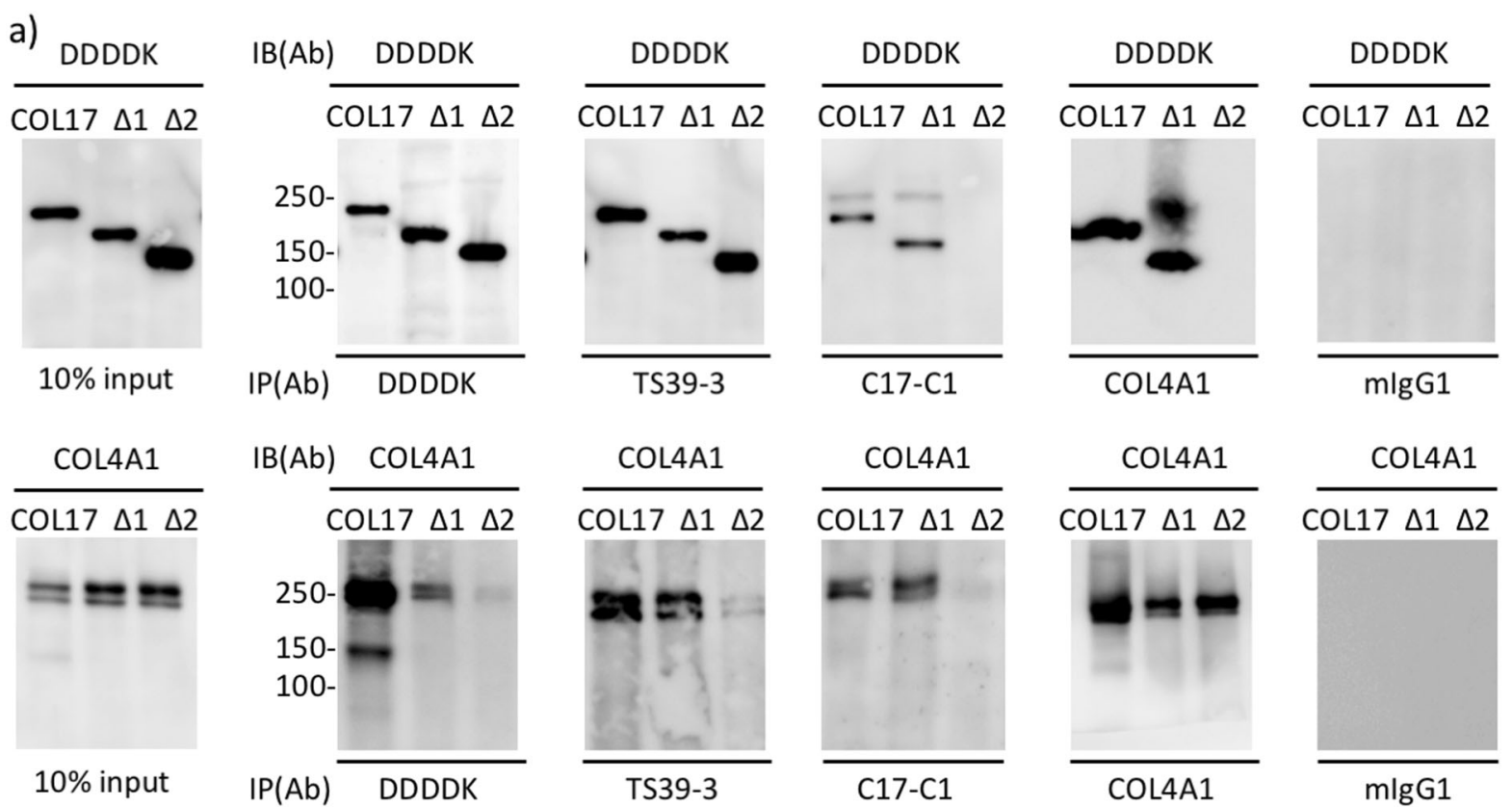

TS39-3
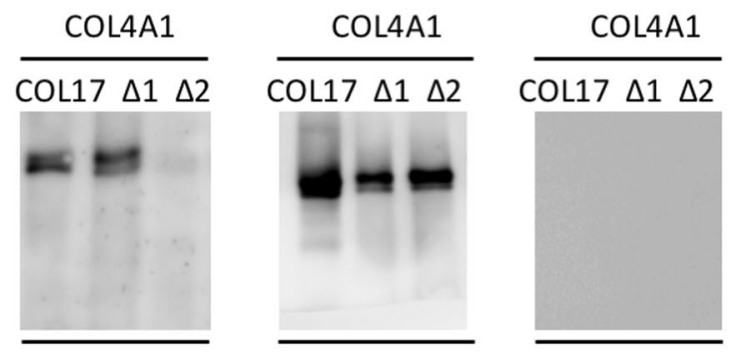

COL4A1

$\mathrm{mlg} G 1$

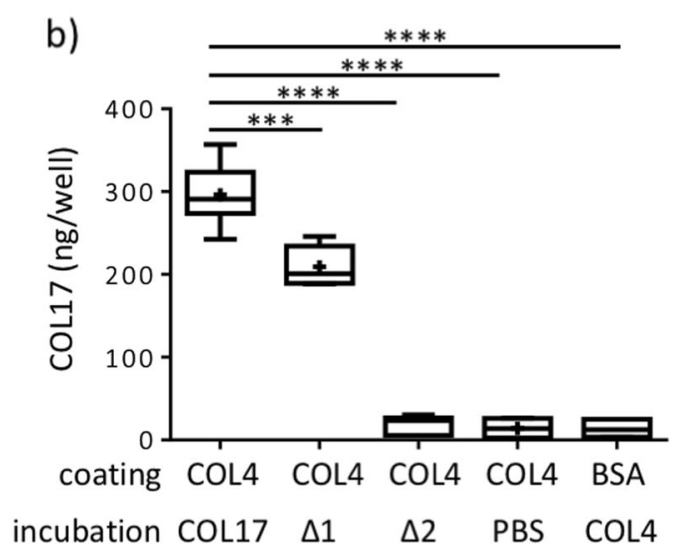

Fig. 3 C-terminus of COL17 directly binds to COL4. a Immunoprecipitation with anti-DDDDK, mAbs TS39-3, C17-C1, or mIgG1 using cell lysates of HEK293 expressing recombinant human COL17 (fulllength $\operatorname{COL} 17, \operatorname{COL} 17 \Delta 1$, and $\operatorname{COL} 17 \Delta 2$ ). Immunoblotting was performed using anti-DDDDK $\mathrm{Ab}$ or anti-human COL4 $\mathrm{Ab}$. The protein amounts in the cell lysates were analyzed by immunoblotting as input (10\% amount of IP). b In vitro binding test between COL17

\section{C-terminus of COL17 directly binds to COL4}

C-terminus of COL17 may bind to COL4 in the oral mucosa. To identify the precise binding region of COL17, we used HEK293 cells stably expressing full-length COL17 or the deletion mutants COL $17 \Delta 1$ and COL17 $\Delta 2$ [5]. The $\mathrm{Met}^{1}$ to $\mathrm{Asp}^{1340}$ amino acid fragment and the $\mathrm{Met}^{1}$ to $\operatorname{Arg}^{1174}$ amino acid fragment of COL17 were designated COL3 and NC6, respectively (Fig. 2a). Full-length COL17, $\operatorname{COL} 17 \Delta 1$, and $\operatorname{COL} 17 \Delta 2$ were precipitated with antiDDDDK mAb (Fig. 3a). In contrast, the amount of COL4 precipitated with anti-DDDDK $\mathrm{mAb}$ decreased with and COL4. The plates were coated with human COL4 protein $(500 \mathrm{ng} /$ well) and then incubated with full-length COL17 (500 ng/well). To quantify COL17 binding amounts to COL4-coated plates, the standard curve was measured by serial diluted full-length human COL17. The data are expressed as the mean \pm S.D. One-way ANOVA. $* * * 0.0001$ $<p<0.001, * * * * p<0.0001$. IP (Ab) immunoprecipitation antibody, IB $(\mathrm{Ab})$ immunoblotting antibody

COL17 $\Delta 1$ and COL17 $\Delta 2$. COL17 $\Delta 2$ was precipitated by mAb TS39-3 but not by mAb C17-C1 due to the epitope of mAb C17-C1 [30] (Fig. 3a). COL4 was clearly detected when full-length COL17 and COL17 $\Delta 1$ were precipitated with mAbs TS39-3 or C17-C1 (Fig. 3a). However, COL4 was faintly detected when $\operatorname{COL} 17 \Delta 2$ was precipitated with mAbs TS39-3 or C17-C1. These results suggested that amino acids $\mathrm{Gly}^{1175}$ to $\mathrm{Asp}^{1340}$ of COL17 mainly bound to COL4. To confirm these findings, we performed in vitro protein-protein binding assays. The amount of COL17 $\Delta 2$ binding to COL4 was significantly reduced by approximately $90 \%$ (Fig. 3b, $p<0.0001$ ), suggesting the direct 

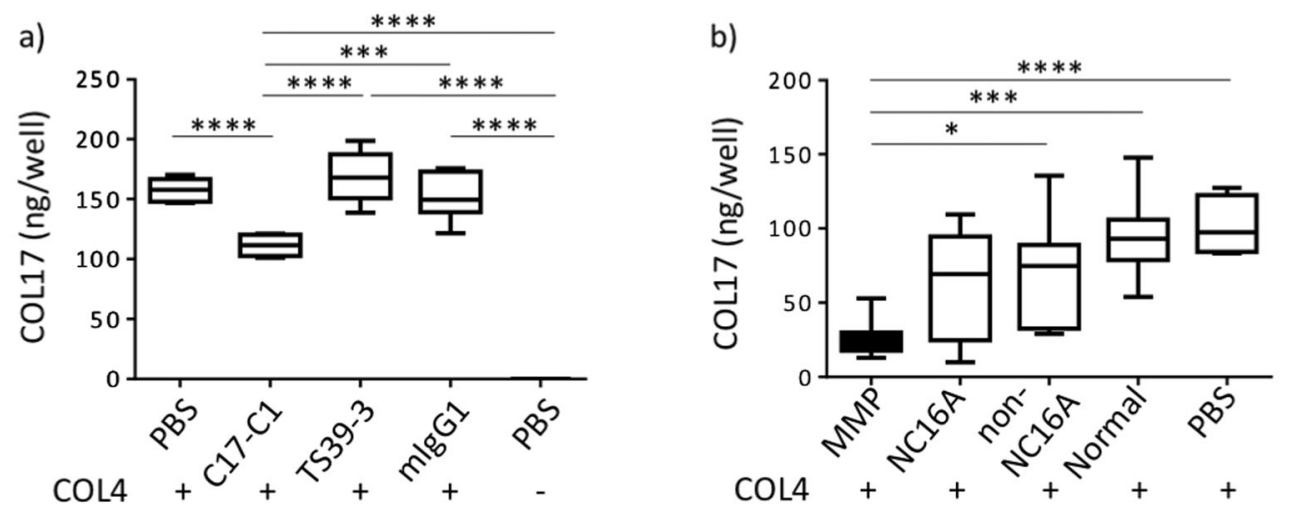

c)

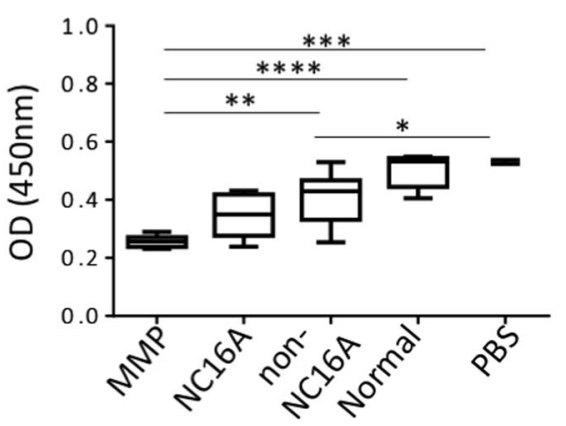

C17-C1

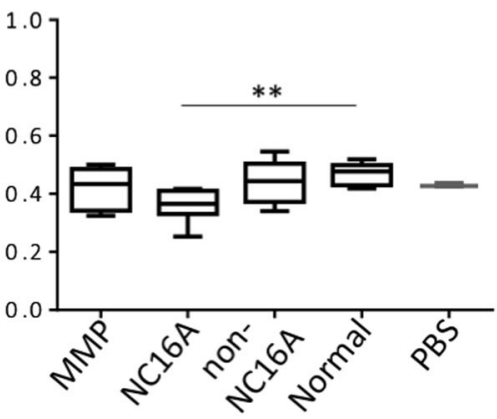

mlgG1

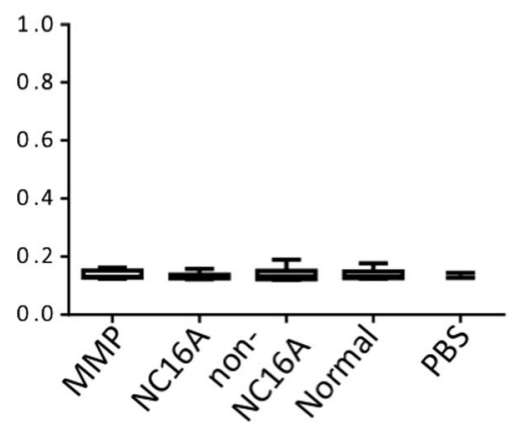

Fig. 4 Autoantibodies targeting the C-terminus of COL17 disrupt the binding between COL17 and COL4. a In vitro binding inhibition test between COL17 and COL4 using mAbs against human COL17. The plates were coated with human COL4 proteins $(500 \mathrm{ng} /$ well). Full-length human COL17 ( $250 \mathrm{ng} /$ well) was preincubated with mAb C17-C1, mAb TS39-3, or mIgG1 (250 ng/well) and then added to the plates. To quantify COL17-binding amounts to COL4-coated plates, the standard curve was measured by serial diluted full-length human COL17. b In vitro binding inhibition test between COL17 and COL4 using MMP-IgG or BP-IgG. MMP-IgG, BP-IgG, or normal human IgG $(20 \mu \mathrm{g} /$ well $)$ was preincubated with full-length

binding of COL4 and COL17, particularly between Gly ${ }^{1175}$ to $\mathrm{Asp}^{1340}$ of COL17.

\section{Autoantibodies targeting the C-terminus of COL17 disrupt the binding between COL17 and COL4}

The present data indicate that the binding region of COL17 with COL4 contains the epitope of $\mathrm{mAb} \mathrm{C} 17-\mathrm{C} 1$. We performed in vitro protein-protein binding assays in the presence of mAbs TS39-3, C17-C1, and normal mouse IgG1 (mIgG1). When full-length COL17 was preincubated with mAb TS39-3 or mIgG1, the amount of COL17 binding to COL4 was unchanged (Fig. 4a). In contrast, preincubation with mAb C17-C1 significantly reduced the amount of COL17 binding to COL4 by $70 \%$ (Fig. 4a). The disturbing effects of $\mathrm{mAb} \mathrm{C} 17-\mathrm{C} 1$ on COL17-COL4 binding were dose-dependent (Supplementary Figure 1). Next, MMP-IgG
COL17 and then added to the COL4-coated plates (250 ng/well). To quantify COL17-binding amounts to COL4-coated plates, the standard curve was measured by serial diluted full-length human COL17. c Competitive COL17-ELISA using mAbs against human COL17 and MMP-IgG or BP-IgG. The full-length COL17-coated plates $(250 \mathrm{ng} /$ well) were incubated with MMP-IgG, BP-IgG, or normal human $\mathrm{IgG}$, followed by incubation with $\mathrm{mAb} \mathrm{C} 17-\mathrm{C} 1$, mAbTS39-3, or mIgG1. The data are expressed as the means \pm S.D. One-way ANOVA. $* 0.01<p<0.05, * * 0.001<p<0.01, * * * 0.0001$ $<p<0.001, * * * * p<0.0001$

or BP-IgG (Supplementary Table 2) was preincubated with COL17 before adding onto the COL4-coated plates. The amounts of COL17 binding to COL4 were significantly decreased when the COL4-coated plates were incubated with MMP-IgG (Fig. 4b). In contrast, typical BP-IgG (NC16A) did not show significant inhibition of COL17-COL4 binding. Even non-NC16A BP-IgG did not prevent the binding of COL17-COL4 (Fig. 4b). These results demonstrated that MMP-IgG hinders the binding of COL17 with COL4, similar to $\mathrm{mAb} \mathrm{C} 17-\mathrm{C} 1$. On the other hand, the preincubation with MMP-IgG inhibited the binding of mAb C17-C1 to the ELISA plates (Fig. 4c, left). Furthermore, typical BP-IgG significantly prevented the binding of $\mathrm{mAb} \mathrm{C} 17-\mathrm{C} 1$ and $\mathrm{mAb}$ TS39-3 (Fig. 4c, left and middle). BP patients had polyclonal autoantibodies against not only $\mathrm{NC16A}$ but also regions outside of NC16A due to epitope spreading. None of the mIgG1 bound to COL17 (Fig. 4c, right). 


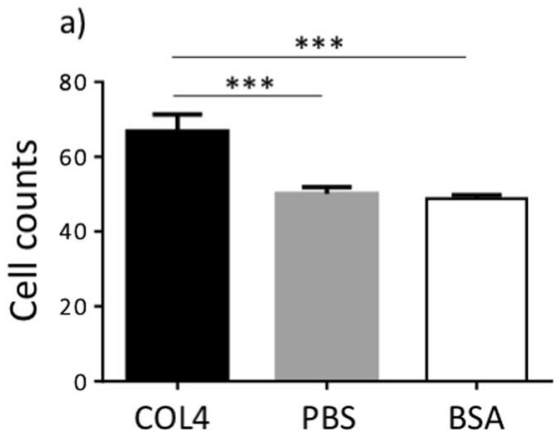

b)

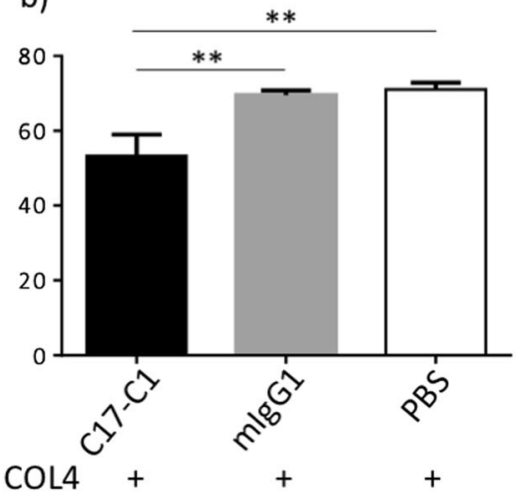

c)

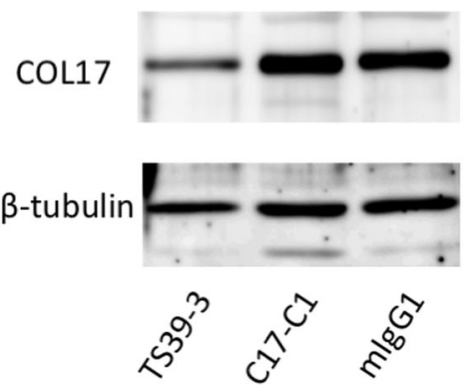

Fig. $5 \mathrm{mAbs}$ targeting the C-terminus of COL17 reduce the cell attachment strength by disrupting the binding of COL17 to COL4 in NHOMKs. a The remaining cell number in the culture plates with or without COL4 coating after vibration stress. b The remaining cell number in COL4-coated plates with the stimulation with mAb C17-C1 or mIgG1 after vibration stress. $\mathbf{c}$ The relative COL17 amounts were evaluated after the 12-h stimulation with mAbs C17-C1, TS39-3, or mIgG1. The data are expressed as the means \pm S.D. One-way ANOVA. $* * 0.001<p<0.01, * * * 0.0001<p<0.001$

in MMP might be dissimilar to that in BP. Hence, we focused on the interaction of COL17 with COL4. The electron microscopy and ECM-binding assay [5, 33] suggested that COL17 interacted with COL4 in the skin. However, the direct binding of these proteins has not been shown. Immunoprecipitaion using $\mathrm{mAb}$ recognizing the NC16A domain or the C-terminus clearly showed that direct COL17-COL4 interactions exist both in NHEKs and NHOMKs. More importantly, only in NHOMKs but not in NHEKs, COL4 was not co-precipitated by mAb C17-C1. These interesting results suggest that the binding region of COL17 to COL4 may differ between in NHEKs and in NHOMKs. Furthermore, the binding may be hindered by mAb C17-C1 in NHOMKs. However, other BMZ components, such as laminin 332 or integrin $\alpha 6 / \beta 4$, may be interposed between COL4 and COL17 and may cause the inhibition by mAb C17-C1 in NHOMKs. This possibility was denied to show that laminin 332 and integrin $\alpha 6 / \beta 4$ were precipitated by both mAbs TS39-3 and C17-C1. Next, we tried to identify the precise region of COL17 interacting with COL4 using recombinant full-length COL17 and Cterminal deleted proteins. In our result, the amount of COL4 precipitated together with COL17 was relatively smaller with $\operatorname{COL} 17 \Delta 2$ than with $\operatorname{COL} 17 \Delta 1$. Considering the results, we suspected that the main binding site of COL17 with COL4 may be between Gly ${ }^{175}$ and Asp ${ }^{1340}$, in which the epitope of $\mathrm{mAb} \mathrm{C} 17-\mathrm{C} 1\left(\mathrm{Gly}^{1316}-\mathrm{Asp}^{1340}\right)$ is located. Furthermore, COL17 may bind to COL4 in more than one domain due to the leap structure of extracellular domain of COL17 [34]. Unlike the results using NHOMKs, mAb C17C1 co-precipitated COL4 with COL17 in NHEK lysates. We could not elucidate the precise regions for COL17-COL4 binding in the skin.

The major targeted epitopes on COL17 are distinct between BP and MMP [20, 21]. Based on previous studies, BP. This observation suggests that the blistering mechanism 
mucosal lesions are associated with autoantibodies against the C-terminus of COL17 in BP and MMP [35]. However, it remains unclear how autoantibodies targeting different epitopes induce diverse clinical manifestations. Regarding the blistering mechanisms in oral lesions, we speculated that autoantibodies in MMP, which often target the C-terminus of COL17 [21], may inhibit the protein-protein interaction in the oral mucosa and reduce hemidesmosomal adhesions. The COL17-COL4 binding was inhibited by $\mathrm{mAb} \mathrm{C} 17-\mathrm{C} 1$ targeting the C-terminus of COL17. This is a similar finding to pemphigus. It is reported that a pathogenic mAb against desmoglein 3 ( $\mathrm{mAb}$ AK23) causes direct inhibition of desmoglein 3 interactions (steric hindrance) [36]. In the present study, we showed that MMP-IgG hinders the binding of COL17 with COL4, similar to mAb C17-C1. Furthermore, MMP autoantibodies share the epitope of mAb C17-C1 by competitive COL17-ELISA. Finally, we demonstrated that the cell adhesion strength was reduced by the treatment of mAb C17-C1 in NHOMKs. Based on our results, we hypothesize the blistering mechanisms; autoantibodies against $\mathrm{C}$-terminus of COL17 hinder the binding of COL17 and COL4 in oral mucosa.

The autoantigens of MMP are reported to be not merely COL17 but also laminin 332, integrin $\alpha 6 \beta 4$, and collagen VII [15]. Recently, an MMP mouse model was reported in which the disease was induced by injection with anti-mouse laminin $\alpha 3$ IgG [37]. Mice injected with IgG developed erosions and crusts not just on the skin but also on the conjunctival, oral, and pharyngeal mucosa. The clinical manifestation of this MMP mouse model was Fc receptordependent and complement-dependent, similar to previous BP mouse models [38-40]. In contrast, non-inflammatory mechanisms of BP were also proposed [41]. BP patient IgG against NC16A significantly induces COL17 depletion in cultured keratinocytes and BP patient skin. COL17 depletion is important for the blistering along the lamina densa without inflammation. Compared to NC16A-IgG, IgG targeting the C-terminus, including MMP-IgG, does not induce COL17 depletion. Our results suggest that the blistering mechanisms in COL17-type MMP are distinctive from those in BP. A recent study revealed that BP patients with normal eosinophil counts presented mucous lesions more frequently than those with higher eosinophila [42]. Furthermore, oral mucosal lesions in some pemphigoid patients were associated with the administration of DPP-4i $[43,44]$. A certain HLA allele (HLA-DQB $1 * 03: 01$ ) was associated with a high risk of DDP-4i BP and MMP occurrence [24, 25]. In light of this evidence, oral mucosal lesions in pemphigoid may be related to be less inflammatory associated with a certain HLA allele.

To summarize, we revealed that COL17 directly binds to COL4 at the BMZ in the keratinocytes. In addition, we provide a novel finding that $\mathrm{mAb}$ targeting the $\mathrm{C}$-terminus of COL17 or MMP-IgG disrupts the binding of COL17 and COL4 in NHOMKs. Pemphigoid IgG targeting the Cterminus of COL17 may play a pathogenic role in the blistering formation in the oral mucosa to inhibit protein-protein interactions with less inflammation.

Acknowledgements We would like to sincerely thank Ms. Mika Tanabe for her technical assistance. This work was supported in part by a JSPS Grant-in-Aid for Scientific Research (C) (16K10141 to HI).

\section{Compliance with ethical standards}

Ethics Statement All studies conformed to the guidelines of the medical ethics committee of Hokkaido University and the Declaration of Helsinki Principles. Written informed consent was obtained before any samples were collected. A full review and approval by an ethics committee of Hokkaido University were not required, according to local guidelines. The studies were conducted in accordance with the Helsinki guidelines.

Conflict of interest The authors declare that they have no conflict of interest.

\section{References}

1. Walko G, Castañón MJ, Wiche G. Molecular architecture and function of the hemidesmosome. Cell Tissue Res. 2015;360:363-78.

2. Goletz S, Zillikens D, Schmidt E. Structural proteins of the dermal-epidermal junction targeted by autoantibodies in pemphigoid diseases. Exp Dermatol. 2017;12:1154-62.

3. Hopkinson SB, Jones JC. The $\mathrm{N}$ terminus of the transmembrane protein BP180 interacts with the N-terminal domain of BP230, thereby mediating keratin cytoskeleton anchorage to the cell surface at the site of the hemidesmosome. Mol Biol Cell. 2000; $11: 277-86$

4. Koster J, Geerts D, Favre B, et al. Analysis of the interactions between BP180, BP230, plectin and the integrin alpha6beta4 important for hemidesmosome assembly. J Cell Sci. 2003;116:387-99.

5. Nishie W, Kiritsi D, Nyström A, et al. Dynamic interactions of epidermal collagen XVII with the extracellular matrix: laminin 332 as a major binding partner. Am J Pathol. 2011;179:829-37.

6. Natsuga $K$, Nishie W, Nishimura M, et al. Loss of interaction between plectin and type XVII collagen results in epidermolysis bullosa simplex. Hum Mutat. 2017;38:1666-70.

7. Hopkinson SB, Findlay K, deHart GW, Jones JC. Interaction of BP180 (type XVII collagen) and alpha6 integrin is necessary for stabilization of hemidesmosome structure. J Invest Dermatol. 1998;111:1015-22.

8. Ishiko A, Shimizu H, Kikuchi A, et al. Human autoantibodies against the 230-kD bullous pemphigoid antigen (BPAG1) bind only to the intracellular domain of the hemidesmosome, whereas those against the $180-\mathrm{kD}$ bullous pemphigoid antigen (BPAG2) bind along the plasma membrane of the hemidesmosome in normal human and swine skin. J Clin Invest. 1993;91:1608-15.

9. Aho S, Uitto J. Direct interaction between the intracellular domains of bullous pemphigoid antigen 2 (BP180) and beta 4 integrin, hemidesmosomal components of basal keratinocytes. Biochem Biophys Res Commun. 1998;243:694-9.

10. Hamill KJ, Hopkinson SB, Jonkman MF, Jones JCR. Type XVII collagen regulates lamellipod stability, cell motility, and signaling 
to Rac1 by targeting bullous pemphigoid antigen 1e to alpha6beta4 integrin. J Biol Chem. 2011;286:26768-80.

11. McGrath JA, Gatalica B, Christiano AM, et al. Mutations in the $180-\mathrm{kD}$ bullous pemphigoid antigen (BPAG2), a hemidesmosomal transmembrane collagen (COL17A1), in generalized atrophic benign epidermolysis bullosa. Nat Genet. 1995;11:83-6.

12. Fine J-D, Bruckner-Tuderman L, Eady RAJ, et al. Inherited epidermolysis bullosa: updated recommendations on diagnosis and classification. J Am Acad Dermatol. 2014;70:1103-26.

13. Schmidt E, Zillikens D. Pemphigoid diseases. Lancet. 2013;381:320-32.

14. Liu Y, Li L, Xia Y. BP180 is critical in the autoimmunity of bullous pemphigoid. Front Immunol. 2017;8:1752.

15. Amber KT, Murrell DF, Schmidt E, et al. Autoimmune subepidermal bullous diseases of the skin and mucosae: clinical features, diagnosis, and management. Clin Rev Allergy Immunol. 2017;381:320-6.

16. Tsunoda K, Ota T, Aoki M, et al. Induction of pemphigus phenotype by a mouse monoclonal antibody against the aminoterminal adhesive interface of desmoglein 3. J Immunol. 2003;170:2170-8.

17. Ishii K, Harada R, Matsuo I, et al. In vitro keratinocyte dissociation assay for evaluation of the pathogenicity of antidesmoglein $3 \mathrm{IgG}$ autoantibodies in pemphigus vulgaris. J Invest Dermatol. 2005;124:939-46.

18. Iwata H, Bieber K, Hirose M, Ludwig RJ. Animal models to investigate pathomechanisms and evaluate novel treatments for autoimmune bullous dermatoses. Curr Pharm Des. 2015;21:2422-39.

19. Ludwig RJ, Vanhoorelbeke K, Leypoldt F, et al. Mechanisms of autoantibody-induced pathology. Front Immunol. 2017;8:603.

20. Di Zenzo G, Grosso F, Terracina M, et al. Characterization of the anti-BP180 autoantibody reactivity profile and epitope mapping in bullous pemphigoid patients. J Invest Dermatol. 2004;122:103-10.

21. Schmidt E, Skrobek C, Kromminga A, et al. Cicatricial pemphigoid: IgA and IgG autoantibodies target epitopes on both intraand extracellular domains of bullous pemphigoid antigen $180 . \mathrm{Br} \mathrm{J}$ Dermatol. 2001;145:778-83.

22. Kamaguchi M, Iwata $H$, Ujiie $H$, et al. High expression of collagen XVII compensates for its depletion induced by pemphigoid IgG in the oral mucosa. J Invest Dermatol. 2018;138:1707-15.

23. Izumi K, Nishie W, Mai Y, et al. Autoantibody profile differentiates between inflammatory and noninflammatory bullous pemphigoid. J Invest Dermatol. 2016;136:2201-10.

24. Ujiie H, Muramatsu K, Mushiroda T, et al. HLA-DQB1*03:01 as a biomarker for genetic susceptibility to bullous pemphigoid induced by DPP-4 inhibitors. $J$ Invest Dermatol. 2017;138:1201-4.

25. Ahmed AR, Foster S, Zaltas M, et al. Association of DQw7 (DQB $1 * 0301)$ with ocular cicatricial pemphigoid. Proc Natl Acad Sci. 1991;88:11579-82.

26. Kamaguchi M, Iwata H, Ujiie I, et al. Direct immunofluorescence using non-lesional buccal mucosa in mucous membrane pemphigoid. Front Med. 2018;5:20.

27. Gammon WR, Fine JD, Forbes M, Briggaman RA. Immunofluorescence on split skin for the detection and differentiation of basement membrane zone autoantibodies. J Am Acad Dermatol. 1992;27:79-87.

28. Toyonaga E, Nishie W, Izumi K, et al. C-terminal processing of collagen XVII induces neoepitopes for linear IgA dermatosis autoantibodies. J Invest Dermatol. 2017;137:2252-559.

29. Ujiie H, Sasaoka T, Izumi K, et al. Bullous pemphigoid autoantibodies directly induce blister formation without complement activation. J Immunol. 2014;193:4415-28.

30. Wada M, Nishie W, Ujiie H, et al. Epitope-dependent pathogenicity of antibodies targeting a major bullous pemphigoid autoantigen collagen XVII/BP180. J Invest Dermatol. 2016;136:938-46.

31. Iwata $\mathrm{H}$, Kitajima $\mathrm{Y}$, Kamio $\mathrm{N}$, et al. IgG from patients with bullous pemphigoid depletes cultured keratinocytes of the 180$\mathrm{kDa}$ bullous pemphigoid antigen (type XVII collagen) and weakens cell attachment. J Invest Dermatol. 2009;129:919-26.

32. Imafuku K, Iwata H, Kamaguchi M, et al. Autoantibodies of noninflammatory bullous pemphigoid hardly deplete type XVII collagen of keratinocytes. Exp Dermatol. 2017;381:320.

33. Nonaka S, Ishiko A, Masunaga T, et al. The extracellular domain of BPAG2 has a loop structure in the carboxy terminal flexible tail in vivo. J Invest Dermatol. 2000;115:889-92.

34. Masunaga $\mathrm{T}$, Shimizu $\mathrm{H}$, Yee $\mathrm{C}$, et al. The extracellular domain of BPAG2 localizes to anchoring filaments and its carboxyl terminus extends to the lamina densa of normal human epidermal basement membrane. J Invest Dermatol. 1997;109:200-6.

35. Hofmann S, Thoma-Uszynski S, Hunziker T, et al. Severity and phenotype of bullous pemphigoid relate to autoantibody profile against the $\mathrm{NH} 2$ - and $\mathrm{COOH}$-terminal regions of the BP180 ectodomain. J Invest Dermatol. 2002;119:1065-73.

36. Saito M, Stahley SN, Caughman CY, et al. Signaling dependent and independent mechanisms in pemphigus vulgaris blister formation. PLoS ONE. 2012;7:e50696.

37. Heppe EN, Tofern S, Schulze FS, et al. Experimental laminin 332 mucous membrane pemphigoid critically involves C5aR1 and reflects clinical and immunopathological characteristics of the human disease. J Invest Dermatol. 2017;137:1709-18.

38. Liu Z, Giudice GJ, Swartz SJ, et al. The role of complement in experimental bullous pemphigoid. J Clin Invest. 1995;95:1539-44.

39. Liu Z, Giudice GJ, Zhou X, et al. A major role for neutrophils in experimental bullous pemphigoid. $\mathrm{J}$ Clin Invest. 1997;100:1256-63.

40. Schulze FS, Beckmann T, Nimmerjahn F, et al. Fcy receptors III and IV mediate tissue destruction in a novel adult mouse model of bullous pemphigoid. Am J Pathol. 2014;184:2185-96.

41. Iwata $H$, Ujiie H. Complement-independent blistering mechanisms in bullous pemphigoid. Exp Dermatol. 2017;26:1235-9.

42. Kridin K. Peripheral eosinophilia in bullous pemphigoid: prevalence and influence on the clinical manifestation. Br J Dermatol. 2018. https://doi.org/10.1111/bjd.16679.

43. Chijiwa C, et al. Decrease in eosinophils infiltrating into the skin of patients with dipeptidyl peptidase-4 inhibitor-related bullous pemphigoid. J Dermatol. 2018;45:596-9.

44. Gaudin O, et al. Gliptin accountability in mucous membrane pemphigoid induction in 24 out of 313 patients. Front Immunol. 2018;9:1030. 